Bull. Austral. Math. Soc.

Vol. 51 (1995) [421-432]

\title{
THE COHOMOLOGY RINGS OF FINITE GROUPS WITH SEMI-DIHEDRAL SYLOW 2-SUBGROUPS
}

\author{
ANgELINA Chin
}

In this paper we determine the mod-2 cohomology rings and the 2-primary part of the integral cohomology rings of finite groups with semi-dihedral Sylow 2subgroups. The method used here is algebraic and can be considered as elementary.

\section{INTRODUCTION}

In this paper we determine the mod-2 cohomology rings and the 2-primary part of the integral cohomology rings of finite groups with semi-dihedral Sylow 2-subgroups. The mod-2 cohomology rings of such groups have in fact been obtained by Martino [4] via topological methods. The method used here is algebraic and can be considered as elementary.

Let $G$ be a finite group with a semi-dihedral Sylow 2-subgroup. The starting point here is a result of Webb [5] on the Poincaré series of $H^{*}\left(G, \mathbb{F}_{2}\right)$. For any subgroup $H$ of $G$, let $A_{G}(H)=N_{G}(H) / C_{G}(B)$ where as usual, $N_{G}(H)$ denotes the normaliser of $H$ in $G$ and $C_{G}(H)$ denotes the centraliser of $H$ in $G$. Using some structural properties of Mackey functors, Webb proved the following:

Theorem. (Webb, [5]) The Poincaré series of $H^{*}\left(G, \mathbb{F}_{2}\right)$ is

$$
P_{G}(t)=\frac{1+t^{5}}{\left(1-t^{3}\right)\left(1-t^{4}\right)}+\frac{\lambda t(1+t)}{\left(1-t^{4}\right)}+\frac{\mu t}{(1-t)\left(1-t^{3}\right)}
$$

where

$$
\lambda=\left\{\begin{array}{ll}
0 & \text { if } 3|| A_{G}\left(Q_{B}\right) \mid \\
1 & \text { otherwise }
\end{array} \text { and } \mu= \begin{cases}0 & \text { if } 3|| A_{G}\left(C_{2} \times C_{2}\right) \mid \\
1 & \text { otherwise. }\end{cases}\right.
$$

The Method

It is well-known that if $A$ is a $G$-ring, the p-part of the cohomology $H^{*}(G, A)_{p}$ is isomorphic (via the restriction map) to the stable elements of the cohomology ring of its Sylow $p$-subgroup for each prime $p$ dividing the order of $G$. Using this fact, the Poincare series of $H^{*}\left(G, \mathbb{F}_{2}\right)$, the ring structure of $H^{*}\left(S D, \mathrm{~F}_{2}\right)$ and the action of the Steenrod algebra on $H^{*}\left(S D, \mathbb{F}_{2}\right)$, we are able to determine the ring structure of $H^{*}\left(G, \mathbf{F}_{2}\right)$.

Receired 28th June, 1994.

Copyright Clearance Centre, Inc. Serial-fee code: 0004-9729/95 \$A2.00+0.00. 


\section{The RING $H^{*}\left(G, \mathbb{F}_{2}\right)$}

The algebra $H^{*}\left(S D, \mathbb{F}_{2}\right)$ has been obtained by Priddy and Evens [3] and is as follows:

$H^{*}\left(S D, \mathbb{F}_{2}\right)$ is generated by the elements

$$
w_{x}, w_{1}, w_{3}, c_{4}
$$

where $\operatorname{deg} w_{x}=\operatorname{deg} w_{1}=1, \operatorname{deg} w_{3}=3, \operatorname{deg} c_{4}=4$ subject to the relations

$$
\begin{array}{ll}
w_{1}^{2}=w_{x} w_{1}, & w_{x}^{2} w_{1}=0, \\
w_{3}^{2}=w_{x}\left(w_{x}+w_{1}\right) c_{4}, & w_{1} w_{3}=0 .
\end{array}
$$

The action of the Steenrod algebra on $H^{*}\left(S D, \mathbb{F}_{2}\right)$ is given as follows:

\begin{tabular}{c|cccc} 
& $\mathrm{Sq}^{1}$ & $\mathrm{Sq}^{2}$ & $\mathrm{Sq}^{3}$ & $\mathrm{Sq}^{4}$ \\
\hline$w_{x}$ & $w_{x}^{2}$ & 0 & 0 & 0 \\
$w_{1}$ & $w_{1}^{2}$ & 0 & 0 & 0 \\
$w_{3}$ & 0 & $\left(w_{x}+w_{1}\right)\left(c_{4}+w_{x} w_{3}\right)$ & $w_{3}^{2}$ & 0 \\
$c_{4}$ & 0 & $w_{x}\left(w_{x}+w_{1}\right) c_{4}$ & 0 & $c_{4}^{2}$
\end{tabular}.

By Webb's theorem, we have four cases to consider.

Case 1. 3||$A_{G}\left(Q_{8}\right)|, 3|\left|A_{G}\left(C_{2} \times C_{2}\right)\right|$.

In this case, the Poincaré series of $H^{*}\left(G, \mathbb{F}_{2}\right)$ is

$$
P_{G}(t)=\frac{1+t^{5}}{\left(1-t^{3}\right)\left(1-t^{4}\right)}
$$

We have

\begin{tabular}{|c|c|c|c|c|c|c|c|c|c|c|c|c|}
\hline$n$ & 1 & 2 & 3 & 4 & 5 & 6 & 7 & 8 & 9 & 10 & 11 & 12 \\
\hline $\operatorname{dim}_{\mathbf{F}_{2}} H^{n}\left(G, \mathbb{F}_{2}\right)$ & 0 & 0 & 1 & 1 & 1 & 1 & 1 & 2 & 2 & 1 & 2 & 3 \\
\hline
\end{tabular}

Let $\alpha, \beta, \mu$ denote the generators of degrees $3,4,5$ in $H^{*}\left(G, \mathbb{F}_{2}\right)$, respectively. The possibilities for the restrictions of these generators to $H^{*}\left(S D, \mathbb{F}_{2}\right)$ are as follows:

\begin{tabular}{|l|l|}
\hline & \multicolumn{1}{|c|}{ The possibilities } \\
\hline $\operatorname{Res}_{G, S D} \alpha$ & $w_{x}^{3}, w_{3}, w_{x}^{3}+w_{3}$ \\
\hline $\operatorname{Res}_{G, S D} \beta$ & $w_{x}^{4}, w_{x} w_{9}, c_{4}, w_{x}^{4}+w_{x} w_{3}, w_{x}^{4}+c_{4}, w_{x} w_{3}+c_{4}$, \\
& $w_{x}^{4}+w_{x} w_{3}+c_{4}$ \\
\hline $\operatorname{Res}_{G, S D} \mu$ & $w_{x}^{5}, w_{x}^{2} w_{3}, w_{x} c_{4}, w_{1} c_{4}, w_{x}^{5}+w_{x}^{2} w_{3}, w_{x}^{5}+w_{x} c_{4}$, \\
& $w_{x}^{5}+w_{1} c_{4}, w_{x}^{2} w_{3}+w_{x} c_{4}, w_{x}^{2} w_{3}+w_{1} c_{4}, w_{x} c_{4}+w_{1} c_{4}$, \\
& $w_{x}^{5}+w_{x}^{2} w_{3}+w_{x} c_{4}, w_{x}^{5}+w_{x}^{2} w_{9}+w_{1} c_{4}, w_{x}^{5}+\left(w_{x}+w_{1}\right) c_{4}$, \\
& $w_{x}^{2} w_{3}+\left(w_{x}+w_{1}\right) c_{4}, w_{x}^{5}+w_{x}^{2} w_{9}+\left(w_{x}+w_{1}\right) c_{4}$ \\
\hline
\end{tabular}

For all the above possibilities, we have that $\alpha^{2} \beta$ and $\mu^{2}$ are both non-zero. Then since $\operatorname{dim}_{F_{2}} H^{10}\left(G, F_{2}\right)=1$, we must have that $\mu^{2}=\alpha^{2} \beta$. From this relation it is clear that $\operatorname{Res}_{G, S D} \mu \neq w_{1} c_{4}$. Therefore $\alpha, \beta, \mu$ are non-nilpotent elements. 
Proposition 1.1. In case $1, \operatorname{Res}_{G, S D} \alpha=w_{9}, \operatorname{Res}_{G, S D} \beta=c_{4}+w_{z}^{4}, \operatorname{Res}_{G, S D} \mu$ $=w_{x}^{2} w_{3}+\left(w_{x}+w_{1}\right) c_{4}$.

Proof: Suppose that $\operatorname{Res}_{G, S D} \alpha=w_{x}^{3}$. Then

$$
\operatorname{Res}_{G, S D} \operatorname{Sq}^{1}(\alpha)=\mathrm{Sq}^{1}\left(w_{x}^{3}\right)=w_{x}^{4} \neq 0
$$

Hence, $\mathrm{Sq}^{1}(\alpha) \neq 0$ and it follows that $\beta=\mathrm{Sq}^{1}(\alpha)$. Since

$$
\operatorname{Res}_{G, S D} \operatorname{Sq}^{2}(\alpha)=\operatorname{Sq}^{2} \operatorname{Res}_{G, S D}(\alpha)=\operatorname{Sq}^{2}\left(w_{x}^{3}\right)=w_{x}^{5} \neq 0
$$

so $\mathrm{Sq}^{2}(\alpha) \neq 0$ and we must have that $\mu=\mathrm{Sq}^{2}(\alpha)$. Therefore

$$
\operatorname{Res}_{G, S D} \beta^{2}=w_{z}^{8}=\operatorname{Res}_{G, S D} \alpha \mu
$$

and hence, $\beta^{2}=\alpha \mu$. Then since $\operatorname{dim}_{\mathrm{F}_{3}} H^{8}\left(G, \mathbb{F}_{2}\right)=2$, there must be an element $\zeta \in H^{8}\left(G, \mathbb{F}_{2}\right)$ such that $H^{8}\left(G, \mathbb{F}_{2}\right)=\left\langle\beta^{2}, \zeta\right\rangle$. Clearly, $\operatorname{Res} G, S D \zeta \neq w_{x}^{8}$. Since $\operatorname{dim}_{\mathbb{F}_{2}} H^{10}\left(G, \mathbb{F}_{2}\right)=1$, so $\operatorname{Res}_{G, S D} \zeta$ must be $c_{4}^{2}$. Then $H^{11}\left(G, \mathbb{F}_{2}\right)=\left\langle\alpha \beta^{2}, \alpha \zeta\right\rangle$ and by inspection, $H^{9}\left(G, \mathbb{F}_{2}\right)=\left\langle\alpha^{3}\right\rangle$; contradicting the fact that $\operatorname{dim}_{\mathbb{F}_{2}} H^{9}\left(G, \mathbb{F}_{2}\right)=2$. Hence, $\operatorname{Res}_{G, S D} \alpha \neq w_{x}^{3}$.

Next suppose that $\operatorname{Res}_{G, S D} \alpha=w_{x}^{3}+w_{3}$. Then

$$
\operatorname{Res}_{G, S D} \operatorname{Sq}^{1}(\alpha)=\operatorname{Sq}^{1} \operatorname{Res}_{G, S D}(\alpha)=S q^{1}\left(w_{x}^{3}+w_{3}\right)=w_{x}^{4} \neq 0
$$

Hence $\operatorname{Sq}^{1}(\alpha) \neq 0$ and so $\operatorname{Sq}^{1}(\alpha)=\beta$. Then $\operatorname{Res}_{G, S D}\left(\mu^{2}\right)=\operatorname{Res}_{G, S D} \alpha^{2} \beta=$ $\left(w_{x}^{6}+w_{3}^{2}\right) w_{x}^{4}=\left(w_{x}^{5}+w_{3} w_{x}^{2}\right)^{2}$. Since $u^{2} \neq 0$ for any non-zero $u \in H^{5}\left(S D, \mathbb{F}_{2}\right)$, it follows that $\operatorname{Res}_{G, S D} \mu=w_{x}^{5}+w_{3} w_{z}^{2}$. Therefore $\operatorname{Res}_{G, S D} \operatorname{Sq}^{1}(\mu)=\operatorname{Sq}^{1}\left(\operatorname{Res}_{G, S D}(\mu)\right)=$ $\mathrm{Sq}^{1}\left(w_{x}^{5}+w_{3} w_{x}^{2}\right)=w_{x}^{6}$. But $w_{x}^{6}$ is not in the image of $\operatorname{Res}_{G, S D}$ generated by $w_{x}^{3}+w_{3}, w_{x}^{4}$ and $w_{x}^{5}+w_{3} w_{x}^{2}$. Hence $\operatorname{Res}_{G, S D} \alpha \neq w_{x}^{3}+w_{3}$.

We must therefore have $\operatorname{Res}_{G, S D} \alpha=w_{3}$. Then $\operatorname{Res}_{G, S D} \operatorname{Sq}^{2}(\alpha)=\operatorname{Sq}^{2} \operatorname{Res}_{G, S D}(\alpha)$ $=\mathrm{Sq}^{2} w_{3}=\left(w_{x}+w_{1}\right)\left(c_{4}+w_{x} w_{3}\right) \neq 0$. Therefore $\operatorname{Sq}^{2}(\alpha) \neq 0$ and hence, $\mu=\operatorname{Sq}^{2}(\alpha)$. Since $\mu^{2}=\alpha^{2} \beta$, so

$$
\begin{aligned}
\left(w_{x}^{2} w_{3}+\left(w_{x}+w_{1}\right) c_{4}\right)^{2} & =\operatorname{Res}_{G, S D} \mu^{2}=\operatorname{Res}_{G, S D}\left(\alpha^{2} \beta\right) \\
& =w_{3}^{2} \operatorname{Res}_{G, S D} \beta
\end{aligned}
$$

Hence, we must have that $\operatorname{Res}_{G, S D} \beta=c_{4}+w_{z}^{4}$.

By Proposition 1.1 and the Poincaré series of $H^{*}\left(G, \mathbb{F}_{2}\right)$ we see that the elements $\alpha, \beta, \mu$ are sufficient to generate the ring $H^{*}\left(G, F_{2}\right)$. We thus have 
Proposition 1.2. In case 1 ,

$$
H^{*}\left(G, \mathbb{F}_{2}\right) \cong \mathbb{F}_{2}[\alpha, \beta, \mu] /\left(\mu^{2}+\alpha^{2} \beta\right),
$$

where $\operatorname{deg} \alpha=3, \operatorname{deg} \beta=4$ and $\operatorname{deg} \mu=5$.

Using the properties of the Steenrod operations, the action of the Steenrod algebra on $H^{*}\left(S D, \mathbb{F}_{2}\right)$ and the fact that $\mathrm{Sq}^{i}$ commutes with $\operatorname{Res}_{G, S D}$, we obtain the following:

Proposition 1.3. In case 1 , the action of the Steenrod algebra on $H^{*}\left(G, \mathbb{F}_{2}\right)$ is given as follows:

\begin{tabular}{c|ccccc} 
& $S q^{1}$ & $S q^{2}$ & $S q^{3}$ & $S q^{4}$ & $S q^{5}$ \\
\hline$\alpha$ & 0 & $\mu$ & $\alpha^{2}$ & 0 & 0 \\
$\beta$ & 0 & $\alpha^{2}$ & 0 & $\beta^{2}$ & 0 \\
$\mu$ & $\alpha^{2}$ & 0 & 0 & $\alpha^{3}+\beta \mu$ & $\mu^{2}$
\end{tabular}.

CASE 2. $3 \backslash|| A_{G}\left(Q_{8}\right)|, 3|\left|A_{G}\left(C_{2} \times C_{2}\right)\right|$.

The Poincaré series of $H^{*}\left(G, \mathbb{F}_{2}\right)$ in this case is

$$
\begin{aligned}
P_{G}(t) & =\frac{1+t^{5}}{\left(1-t^{3}\right)\left(1-t^{4}\right)}+\frac{t(1+t)}{\left(1-t^{4}\right)} \\
& =\frac{1+t+t^{2}-t^{4}}{\left(1-t^{3}\right)\left(1-t^{4}\right)}
\end{aligned}
$$

We have

\begin{tabular}{|c|c|c|c|c|c|c|c|c|c|c|c|c|c|c|c|c|}
\hline$n$ & 1 & 2 & 3 & 4 & 5 & 6 & 7 & 8 & 9 & 10 & 11 & 12 & 13 & 14 & 15 & 16 \\
\hline $\operatorname{dim}_{\mathbf{r}_{3}} \boldsymbol{H}^{n}\left(G, \mathbb{F}_{2}\right)$ & 1 & 1 & 1 & 1 & 2 & 2 & 1 & 2 & 3 & 2 & 2 & 3 & 3 & 3 & 3 & 3 \\
\hline
\end{tabular}

Let $\xi$ denote the generator of degree 1 in $H^{*}\left(G, \mathbb{F}_{2}\right)$. Then $\operatorname{Res}_{G, S D} \xi$ is $\boldsymbol{w}_{1}$, $w_{1}+w_{z}$ or $w_{z}$. Suppose that $\operatorname{Res}_{G, S D} \xi=w_{x}$. Then $\xi$ is a non-nilpotent generator of $H^{*}\left(G, \mathbb{F}_{2}\right)$. Since $\operatorname{dim}_{\mathbb{F}_{2}} H^{n}\left(G, \mathbb{F}_{2}\right)=1$ for $n=3,4$, it follows that $w_{3}$ and $c_{4}$ are non-stable elements of $H^{*}\left(S D, \mathbb{F}_{2}\right)$. Since $\operatorname{dim}_{\mathbf{F}_{2}} H^{5}\left(G, \mathbb{F}_{2}\right)=2$, there must exist an element $\nu \in H^{5}\left(G, \mathbb{F}_{2}\right)$ such that $H^{5}\left(G, \mathbb{F}_{2}\right)=\left\langle\xi^{5}, \nu\right\rangle$. By inspection the possibilities for $\operatorname{Res}_{G, S D} \nu$ are the same as the possibilities for $\operatorname{Res}_{G, S D} \mu$ in Case 1 except for $w_{x}^{5}$. Then $H^{6}\left(G, \mathbf{F}_{2}\right)=\left\langle\xi^{6}, \xi \nu\right\rangle$. Since $\operatorname{dim}_{\mathbf{F}_{2}} H^{7}\left(G, \mathbb{F}_{2}\right)=1$ and $\xi^{7} \neq 0$, so we must have $\operatorname{Res}_{G, S D} \nu=w_{1} c_{4}$. Since $\operatorname{dim}_{\mathbb{F}_{2}} H^{8}\left(G, \mathbb{F}_{2}\right)=2$ there is some element $\zeta \in H^{8}\left(G, \mathbb{F}_{2}\right)$ such that $H^{8}\left(G, \mathbb{F}_{2}\right)=\left\langle\xi^{8}, \zeta\right\rangle$. For all the possibilities of Res $G, S D \zeta$ we have that $\xi^{10}, \nu^{2}$ and $\xi^{2} \zeta$ are $\mathbb{F}_{2}$-linearly independent; contradicting the fact that $\operatorname{dim}_{\mathbb{F}_{2}} H^{10}\left(G, \mathbf{F}_{2}\right)=2$. By the same argument, $\operatorname{Res}_{G, S D} \xi \neq w_{1}+w_{x}$. Therefore $\operatorname{Res}_{G, S D} \xi=w_{1}$. Since $w_{1}^{3}=0$, so $\xi^{3}=0$. 
Let $\alpha$ and $\beta$ denote the generators of degrees 3 and 4 in $H^{*}\left(G, \mathbb{F}_{2}\right)$, respectively. The possibilities for $\operatorname{Res}_{G, S D} \alpha$ are $w_{x}^{3}, w_{3}$ and $w_{x}^{3}+w_{3}$. Therefore $\operatorname{Res}_{G, S D} \xi \alpha=0$ and hence $\xi \alpha=0$. It follows that $0=\mathrm{Sq}^{1}(\xi \alpha)=\xi^{2} \alpha+\xi \mathrm{Sq}^{1}(\alpha)=\xi \mathrm{Sq}^{1}(\alpha)$. If $\mathrm{Sq}^{1}(\alpha)=\beta$, then $\xi \beta=0$ and the possibilities for $\operatorname{Res}{ }_{G, S D} \beta$ are $w_{x}^{4}, w_{x} w_{3}$ and $w_{x}^{4}+w_{x} w_{3}$. But $\operatorname{Sq}^{1}(\beta)=0$ implies that $\operatorname{Res}_{G, S D} \beta=w_{x}^{4}$ and hence, $\operatorname{Res}_{G, S D} \alpha=w_{x}^{3}$ or $w_{x}^{3}+w_{3}$.

Since $\operatorname{dim}_{\mathbb{T}_{2}} H^{5}\left(G, \mathbb{F}_{2}\right)=2$, there are elements $\mu, \nu$ such that $H^{5}\left(G, \mathbb{F}_{2}\right)=\langle\mu, \nu\rangle$. Then since $\operatorname{dim}_{\mathbb{F}_{2}} H^{10}\left(G, \mathbb{F}_{2}\right)=2$, we must have that

$$
\operatorname{Res}_{G, S D} \alpha=w_{x}^{3}, \operatorname{Res}_{G, S D} \mu=w_{x}^{5} \text { and } \operatorname{Res}_{G, S D} \nu=w_{1} c_{4} .
$$

Then

$$
H^{6}\left(G, \mathbb{F}_{2}\right)=\left\langle\xi \nu, \alpha^{2}\right\rangle \text { and } H^{7}\left(G, \mathbb{F}_{2}\right)=\langle\alpha \beta\rangle \text {. }
$$

Since $\beta^{2}=\alpha \mu \in H^{8}\left(G, \mathbb{F}_{2}\right)$ and $\operatorname{dim}_{\mathrm{F}_{2}} H^{8}\left(G, \mathbb{F}_{2}\right)=2$, there must exist an element $\zeta \in H^{8}\left(G, \mathbb{F}_{2}\right)$ such that $H^{8}\left(G, \mathbb{F}_{2}\right)=\langle\alpha \mu, \zeta\rangle$. In order that $\operatorname{dim}_{\mathbb{F}_{2}} H^{10}\left(G, \mathbb{F}_{2}\right)=2$, we must have $\operatorname{Res}_{G, S D} \zeta=c_{4}^{2}$. Then $H^{9}\left(G, \mathbb{F}_{2}\right)=\left\langle\xi \zeta, \alpha^{3}, \eta\right\rangle$ for some $\eta$.

Since $\operatorname{dim}_{\mathbf{F}_{2}} H^{10}\left(G, \mathbb{F}_{2}\right)=2$, so $\operatorname{Res}_{G, S D} \eta$ must be an $\mathbb{F}_{2}$-linear combination of $w_{x}^{6} w_{3}$ and $w_{x}^{2} w_{3} c_{4}$. Then $\operatorname{Res}_{G, S D} S q^{2} \eta \in\left\langle w_{x}^{7} c_{4}, w_{x}^{4} w_{3} c_{4}+w_{x}^{3} c_{4}^{2}\right\rangle$. Note that $\operatorname{Res}_{G, S D} \alpha \beta^{2}=w_{x}^{11}$ and $\operatorname{Res}_{G, S D} \alpha \zeta=w_{x}^{3} c_{4}^{2}$. Then $\alpha \beta^{2}, \alpha \zeta$ and $S q^{2} \eta$ are $\mathbb{F}_{2}$-linearly independent in $H^{11}\left(G, \mathbb{F}_{2}\right)$; contradicting the fact that $\operatorname{dim}_{\mathbb{F}_{2}} H^{11}\left(G, \mathbb{F}_{2}\right)=2$.

Therefore $\operatorname{Sq}^{1}(\alpha)=0$ and we must have $\operatorname{Res}_{G, S D} \alpha=w_{3}$. By an argument similar to that above we have $\xi \beta \neq 0$, so the possibilities for $\operatorname{Res}_{G, S D} \beta$ are $c_{4}, w_{x}^{4}+c_{4}$, $w_{x} w_{3}+c_{4}$ and $w_{x}^{4}+w_{x} w_{3}+c_{4}$. If $\operatorname{Res}_{G, S D} \beta$ is $w_{x} w_{3}+c_{4}$ or $w_{x}^{4}+w_{x} w_{3}+c_{4}$, then $\operatorname{Res}_{G, S D} \operatorname{Sq}^{1}(\beta)=\operatorname{Sq}^{1} \operatorname{Res}_{G, S D}(\beta)=w_{x}^{2} w_{3}$. Therefore $H^{5}\left(G, \mathbb{F}_{2}\right)=\langle\xi \beta, \mu\rangle$ where $\operatorname{Res}_{G, S D} \mu=w_{x}^{2} w_{3}$. Then $H^{10}\left(G, \mathbb{F}_{2}\right)=\left\langle\mu^{2}, \xi^{2} \beta^{2}, \alpha^{2} \beta\right\rangle$; contradicting the fact that $\operatorname{dim}_{\mathbb{F}_{2}} H^{10}\left(G, \mathbb{F}_{2}\right)=2$. Suppose $\operatorname{Res}_{G, S D} \beta=c_{4}$. Then $H^{5}\left(G, \mathbb{F}_{2}\right)=\langle\xi \beta, \mu\rangle$ for some $\mu$ such that $\operatorname{Res}_{G, S D} \mu \neq w_{1} c_{4}$. Since $\operatorname{Res}_{G, S D} \operatorname{Sq}^{2}(\alpha)=\operatorname{Sq}^{2} \operatorname{Res}_{G, S D} \alpha=$ $\mathrm{Sq}^{2}\left(w_{3}\right)=w_{x}^{2} w_{3}+\left(w_{x}+w_{1}\right) c_{4}$, so $\operatorname{Res}_{G, S D} \mu$ is either $w_{x}^{2} w_{3}+\left(w_{x}+w_{1}\right) c_{4}$ or $w_{x}^{2} w_{3}+w_{x} c_{4}$. In either case $H^{10}\left(G, \mathbb{F}_{2}\right)=\left\langle\xi^{2} \beta^{2}, \alpha^{2} \beta, \mu^{2}\right\rangle$; contradicting the fact that $\operatorname{dim}_{\mathbb{F}_{2}} H^{10}\left(G, \mathbb{F}_{2}\right)=2$. Hence, we must have $\operatorname{Res}_{G, S D} \beta=c_{4}+w_{x}^{4}$. By the same reason, there is some element $\mu$ such that $H^{5}\left(G, \mathbb{F}_{2}\right)=\langle\xi \beta, \mu\rangle$ and $\operatorname{Res}_{G, S D \mu} \mu w_{x}^{2} w_{3}+\left(w_{x}+w_{1}\right) c_{4}$. Then $\xi \mu=0$ and $\mu^{2}=\alpha^{2} \beta$. It is straightforward to check that $\xi, \alpha, \beta$ and $\mu$ are sufficient to generate the ring $H^{*}\left(G, \mathbb{F}_{2}\right)$.

We have thus proved the following:

Proposition 1.4. In case 2,

$$
H^{*}\left(G, \mathbb{F}_{2}\right)=\mathbb{F}_{2}[\xi, \alpha, \beta, \mu] /\left(\xi^{3}, \xi \alpha, \xi \mu, \mu^{2}+\alpha^{2} \beta\right)
$$

where $\operatorname{deg} \xi=1, \operatorname{deg} \alpha=3, \operatorname{deg} \beta=4, \operatorname{deg} \mu=5$,

$$
\operatorname{Res}_{G, S D} \xi=w_{1}, \quad \operatorname{Res}_{G, S D} \alpha=w_{3}, \quad \operatorname{Res}_{G, S D} \beta=c_{4}+w_{x}^{4}
$$


and $\operatorname{Res}_{G, S D} \mu=w_{x}^{2} w_{3}+\left(w_{x}+w_{1}\right) c_{4}$.

Using the Steenrod algebra axioms, we obtain

Proposition 1.5. In case 2 , the action of the Steenrod algebra on $H^{*}\left(G, \mathbb{F}_{2}\right)$ is given as follows:

\begin{tabular}{c|ccccc} 
& $S q^{1}$ & $S q^{2}$ & $S q^{3}$ & $S q^{4}$ & $S q^{5}$ \\
\hline$\xi$ & $\xi^{2}$ & 0 & 0 & 0 & 0 \\
$\alpha$ & 0 & $\mu$ & $\alpha^{2}$ & 0 & 0 \\
$\beta$ & 0 & $\alpha^{2}$ & 0 & $\beta^{2}$ & 0 \\
$\mu$ & $\alpha^{2}$ & 0 & 0 & $\alpha^{3}+\beta \mu$ & $\mu^{2}$
\end{tabular}.

CASE 3. 3||$A_{G}\left(Q_{8}\right)|, 3 \gamma| A_{G}\left(C_{2} \times C_{2}\right) \mid$.

The Poincaré series of $H^{*}\left(G, \mathbb{F}_{2}\right)$ in this case is

$$
\begin{aligned}
P_{G}(t) & =\frac{1+t^{5}}{\left(1-t^{3}\right)\left(1-t^{4}\right)}+\frac{t}{(1-t)\left(1-t^{3}\right)} \\
& =\frac{1+t^{3}}{(1-t)\left(1-t^{4}\right)} .
\end{aligned}
$$

We have

\begin{tabular}{|c|c|c|c|c|c|c|c|c|c|c|c|c|}
\hline$n$ & 1 & 2 & 3 & 4 & 5 & 6 & 7 & 8 & 9 & 10 & 11 & 12 \\
\hline $\operatorname{dim}_{\mathbb{F}_{2}} H^{n}\left(G, \mathbb{F}_{2}\right)$ & 1 & 1 & 2 & 3 & 3 & 3 & 4 & 5 & 5 & 5 & 6 & 7 \\
\hline
\end{tabular}

Let $\xi$ denote the generator of degree 1 in $H^{*}\left(G, \mathbb{F}_{2}\right)$. Then $\operatorname{Res} G, S D \xi$ is one of $w_{1}, w_{x}$ and $w_{1}+w_{x}$. Suppose that $\operatorname{Res}_{G, S D} \xi=w_{1}$. Then

$$
\begin{aligned}
& H^{2}\left(G, \mathbb{F}_{2}\right)=\left\langle\xi^{2}\right\rangle, \\
& H^{3}\left(G, \mathbb{F}_{2}\right)=\left\langle a_{3}, b_{3}\right\rangle
\end{aligned}
$$

where $\operatorname{Res}_{G, S D} a_{3}=w_{3}, \operatorname{Res}_{G, S D} b_{3}=w_{x}^{3}$,

$$
H^{4}\left(G, \mathbb{F}_{2}\right)=\left\langle a_{4}, b_{4}, d_{4}\right\rangle
$$

where $\operatorname{Res}_{G, S D} a_{4}=c_{4}, \operatorname{Res}_{G, S D} b_{4}=w_{x}^{4}, \operatorname{Res}_{G, S D} d_{4}=w_{x} w_{3}$,

$$
H^{5}\left(G, \mathbb{F}_{2}\right)=\left\langle\xi a_{4}, a_{5}, b_{5}, c_{5}\right\rangle
$$

where $\operatorname{Res}_{G, S D} a_{5}=w_{x}^{5}, \operatorname{Res}_{G, S D} b_{5}=w_{x}^{2} w_{3}, \operatorname{Res}_{G, S D} c_{5}=w_{x} c_{4}$; contradicting the fact that $\operatorname{dim}_{\mathbf{F}_{2}} H^{5}\left(G, \mathbb{F}_{2}\right)=3$. 
Now suppose that $\operatorname{Res}_{G, S D} \xi=w_{x}$. Then

$$
\begin{aligned}
& H^{2}\left(G, \mathbb{F}_{2}\right)=\left\langle\xi^{2}\right\rangle, \\
& H^{3}\left(G, \mathbb{F}_{2}\right)=\left\langle\xi^{3}, a_{3}\right\rangle \quad \text { where } \operatorname{Res}_{G, S D} a_{3}=w_{3}, \\
& H^{4}\left(G, \mathbb{F}_{2}\right)=\left\langle\xi^{4}, \xi a_{3}, a_{4}\right\rangle \text { where } \operatorname{Res}_{G, S D} a_{4}=c_{4}, \\
& H^{5}\left(G, \mathbb{F}_{2}\right)=\left\langle\xi^{5}, \xi^{2} a_{3}, \xi a_{4}\right\rangle, \\
& H^{6}\left(G, \mathbb{F}_{2}\right)=\left\langle\xi^{6}, \xi^{3} a_{3}, \xi^{2} a_{4}, a_{3}^{2}\right\rangle
\end{aligned}
$$

contradicting the fact that $\operatorname{dim}_{\mathrm{F}_{2}} H^{6}\left(G, \mathbb{F}_{2}\right)=3$. Hence, we must have $\operatorname{Res}_{G, S D} \xi=$ $w_{x}+w_{1}$. Then

$$
\begin{aligned}
& H^{3}\left(G, \mathbb{F}_{2}\right)=\left\langle\xi^{3}, \mu\right\rangle \text { where } \operatorname{Res}_{G, S D} \mu=w_{3}, \\
& H^{4}\left(G, \mathbb{F}_{2}\right)=\left\langle\xi^{4}, \xi \mu, \beta\right\rangle \quad \text { where } \quad \operatorname{Res}_{G, S D} \beta=c_{4}, \\
& H^{5}\left(G, \mathbb{F}_{2}\right)=\left\langle\xi^{5}, \xi^{2} \mu, \xi \beta\right\rangle .
\end{aligned}
$$

Since $\operatorname{Res}_{G, S D} \mu^{2}=w_{3}^{2}=\left(w_{x}^{2}+w_{1}^{2}\right) c_{4}=\operatorname{Res}_{G, S D} \xi^{2} \beta$, so $\mu^{2}=\xi^{2} \beta$. By inspection, we have that $\xi, \mu, \beta$ are sufficient to generate the ring. We have thus proved the following:

Proposition 1.6. In case 3 ,

$$
H^{*}\left(G, \mathbb{F}_{2}\right)=\mathbb{F}_{2}[\xi, \mu, \beta] /\left(\mu^{2}+\xi^{2} \beta\right)
$$

where $\operatorname{deg} \xi=1, \operatorname{deg} \mu=3, \operatorname{deg} \beta=4$,

$$
\operatorname{Res}_{G, S D} \xi=w_{x}+w_{1}, \quad \operatorname{Res}_{G, S D} \mu=w_{3}, \quad \operatorname{Res}_{G, S D} \beta=c_{4} .
$$

By the Steenrod algebra axioms, we have

Proposition 1.7. In case 3, the action of the Steenrod algebra on $H^{*}\left(G, \mathbb{F}_{2}\right)$ is given as follows:

\begin{tabular}{c|cccc} 
& $S q^{1}$ & $S q^{2}$ & $S q^{3}$ & $S q^{4}$ \\
\hline$\xi$ & $\xi^{2}$ & 0 & 0 & 0 \\
$\mu$ & 0 & $\xi^{2} \mu+\xi \beta$ & $\mu^{2}$ & 0 \\
$\beta$ & 0 & $\mu^{2}$ & 0 & $\beta^{2}$
\end{tabular}.

CASE 4. $3 \not\left|A_{G}\left(Q_{8}\right)\right|, 3 \gamma\left|A_{G}\left(C_{2} \times C_{2}\right)\right|$.

The Poincaré series of $H^{*}\left(G, \mathbb{F}_{2}\right)$ in this case is

$$
\begin{aligned}
P_{G}(t) & =\frac{1+t^{5}}{\left(1-t^{3}\right)\left(1-t^{4}\right)}+\frac{t(1+t)}{1-t^{4}}+\frac{t}{(1-t)\left(1-t^{3}\right)} \\
& =\frac{1+t}{(1-t)\left(1-t^{4}\right)} .
\end{aligned}
$$

This is the same as the Poincare series of $H^{*}\left(S D, \mathbb{F}_{2}\right)$. We thus have 
Proposition 1.8. In case $4, H^{*}\left(G, \mathbb{F}_{2}\right) \cong H^{*}\left(S D, \mathbb{F}_{2}\right)$.

The action of the Steenrod algebra on $H^{*}\left(G, \mathbb{F}_{2}\right)$ follows from the action on $H^{*}\left(S D, \mathbb{F}_{2}\right)$.

\section{REMARK.}

This method can clearly be used to obtain the cohomology ring of a finite group once its additive structure and the cohomology of its Sylow p-subgroups are known.

In [1], Asai and Sasaki obtained the mod-2 cohomology rings of finite groups with dihedral or quaternion Sylow 2-subgroups algebraically. The method used here is different from theirs. We note that one of the criteria for the method of Asai and Sasaki to work is that $\operatorname{dim}_{\mathbb{Y}_{2}} H^{n+1}\left(G, \mathbb{F}_{2}\right) \geqslant \operatorname{dim}_{\mathbb{F}_{2}} H^{n}\left(G, \mathbb{F}_{2}\right)$ for all $n \geqslant 1$. (see [1, Theorem 2.5]). We thus see that their method would not work in general for finite groups with semi-dihedral Sylow 2-subgroups.

\section{ThE 2-PART $H^{*}(G, \mathbb{Z})_{2}$}

Let $\Delta: H^{i}\left(G, \mathbb{F}_{2}\right) \rightarrow H^{i+1}\left(G, \mathbb{F}_{2}\right)$ and $\delta: H^{i}\left(G, \mathbb{F}_{2}\right) \rightarrow H^{i+1}(G, \mathbb{Z})$ denote the Bockstein homomorphisms. Then $\Delta=\pi_{*} \cdot \delta$ where $\pi_{*}: H^{i}(G, \mathbb{Z}) \rightarrow H^{i}\left(G, \mathbb{F}_{2}\right)$ is induced from $\pi: \mathbb{Z} \rightarrow \mathbb{F}_{2}$.

Proposition 2.1. (Cárdenas and Lluis, [2]) The Poincaré series of $H^{*}(G, \mathbb{Z})_{2} \otimes \mathbb{F}_{2}$ is

$$
P_{G}\left(H^{*}(G, \mathbb{Z})_{2} \otimes \mathbb{F}_{2}, t\right)=\frac{t}{1+t} P_{G}\left(H^{*}\left(G, \mathbb{F}_{2}\right), t\right)+\frac{1}{1+t}
$$

The integral cohomology ring of a semi-dihedral group has been obtained by Priddy and Evens [3]:

If $S D$ is of order $2^{n+1}$, then

$$
H^{*}(S D, \mathbb{Z})=\mathbb{Z}[\alpha, \beta, \eta, \gamma]
$$

where $\operatorname{deg} \eta=\operatorname{deg} \beta=2, \operatorname{deg} \alpha=4, \operatorname{deg} \gamma=5$ subject to the relations

$$
\begin{aligned}
2^{n} \alpha & =2 \beta=2 \eta=2 \gamma=0, \\
\eta^{2} & =\eta \beta=\eta \gamma=0, \quad \gamma^{2}=\beta^{3} \alpha \quad(n \geqslant 3) .
\end{aligned}
$$

CASE (1). 3||$A_{G}\left(Q_{8}\right)|, 3|\left|A_{G}\left(C_{2} \times C_{2}\right)\right|$.

By Proposition 2.1,

$$
\begin{aligned}
P_{G}\left(H^{*}(G, \mathbb{Z})_{2} \otimes F_{2}, t\right) & =\frac{1+t^{5}}{\left(1-t^{3}\right)\left(1-t^{4}\right)} \cdot \frac{t}{1+t}+\frac{1}{1+t} \\
& =\frac{1-t^{3}+t^{6}}{\left(1-t^{3}\right)\left(1-t^{4}\right)} .
\end{aligned}
$$


We have

\begin{tabular}{|c|c|c|c|c|c|c|c|c|c|c|c|c|}
\hline$n$ & 1 & 2 & 3 & 4 & 5 & 6 & 7 & 8 & 9 & 10 & 11 & 12 \\
\hline $\operatorname{dim}_{\mathbf{Y}_{2}} H^{n}(G, \mathbb{Z})_{2} \otimes \mathbb{F}_{2}$ & 0 & 0 & 0 & 1 & 0 & 1 & 0 & 1 & 1 & 1 & 0 & 2 \\
\hline
\end{tabular}

Let $\xi$ and $\zeta$ denote the generators of degrees 4 and 6 in $H^{*}(G, \mathbb{Z})_{2}$, respectively. We have shown that $H^{*}\left(G, \mathbb{F}_{2}\right)=\mathbb{F}_{2}\left[a_{3}, b_{4}, c_{5}\right] /\left(c_{5}^{2}+a_{3}^{2} b_{4}\right)$ where the subscript gives the degree of the generator. Since $\Delta\left(a_{3}\right)=\mathrm{Sq}^{1}\left(a_{3}\right)=0$, so $\delta\left(a_{3}\right) \in \operatorname{Ker} \pi_{*}=\operatorname{Im} 2$. Then since $\operatorname{Res}_{G, S D} a_{3}=w_{9}$, so $\delta\left(a_{3}\right)=2^{n-1} \xi$. Since $\Delta\left(c_{5}\right)=a_{3}^{2} \neq 0$, so $\delta\left(c_{5}\right) \neq 0$. We may then take $\zeta=\delta\left(c_{5}\right)$. Let $\nu$ denote the generator of degree 9 in $H^{*}(G, \mathbb{Z})_{2}$. Since $\Delta\left(a_{3} c_{5}\right)=a_{3}^{3} \neq 0$, so $\delta\left(a_{3} c_{5}\right) \neq 0$. Hence, we may take $\nu=\delta\left(a_{3} c_{5}\right)$. Note that

$$
\nu^{2}=\delta\left(a_{3} c_{5}\right) \delta\left(a_{3} c_{5}\right)=\delta\left(\left(a_{3} c_{5}\right) \Delta\left(a_{3} c_{5}\right)\right)=\delta\left(a_{3}^{4} c_{5}\right)
$$

and

$$
\zeta^{3}=\delta\left(c_{5}\right) \delta\left(c_{5}\right)^{2}=\delta\left(c_{5} \Delta\left(c_{5}\right)\right) \delta\left(c_{5}\right)=\delta\left(a_{3}^{2} c_{5}\right) \delta\left(c_{5}\right)=\delta\left(a_{3}^{4} c_{5}\right)
$$

Therefore, $\nu^{2}=\zeta^{3}$. By inspection, the elements $\xi, \zeta$ and $\nu$ are sufficient to generate $H^{*}(G, \mathbb{Z})_{2}$. We have thus shown

Proposition 2.2. In case $1, H^{*}(G, \mathbb{Z})_{2}$ is generated by the elements

$$
\xi, \zeta, \nu
$$

where $\operatorname{deg} \xi=4, \operatorname{deg} \zeta=6, \operatorname{deg} \nu=9$ subject to the relations

$$
2^{n} \xi=2 \zeta=2 \nu=0
$$

and

$$
\nu^{2}=\zeta^{3} \text {. }
$$

Further, $\operatorname{Res}_{G, S D} \xi=\alpha, \operatorname{Res}_{G, S D} \zeta=\alpha \beta$ and $\operatorname{Res}_{G, S D} \nu=\gamma \alpha$.

CASE (2). $3 \nmid|| A_{G}\left(Q_{8}\right)|, 3|\left|A_{G}\left(C_{2} \times C_{2}\right)\right|$.

By Proposition 2.1,

$$
\begin{aligned}
P_{G}\left(H^{*}(G, \mathbb{Z})_{2} \otimes \mathbb{F}_{2}, t\right) & =\frac{1+t+t^{2}-t^{4}}{\left(1-t^{3}\right)\left(1-t^{4}\right)} \cdot \frac{t}{1+t}+\frac{1}{1+t} \\
& =\frac{1+t^{2}-t^{3}-t^{5}+t^{6}}{\left(1-t^{3}\right)\left(1-t^{4}\right)}
\end{aligned}
$$

We have

\begin{tabular}{|c|c|c|c|c|c|c|c|c|c|c|c|c|}
\hline$n$ & 1 & 2 & 3 & 4 & 5 & 6 & 7 & 8 & 9 & 10 & 11 & 12 \\
\hline $\operatorname{dim}_{\mathbb{F}_{2}} H^{n}(G, \mathbb{Z})_{2} \otimes \mathbf{F}_{2}$ & 0 & 1 & 0 & 1 & 0 & 2 & 0 & 1 & 1 & 2 & 0 & 2 \\
\hline
\end{tabular}


For convenience, let $\xi=a_{1}, \alpha=b_{3}, \beta=d_{4}, \mu=e_{5}$ in Proposition 1.4. Let $\xi$ denote the generator of degree 2 in $H^{*}(G, \mathbb{Z})_{2}$. Since $\Delta\left(a_{1}\right)=a_{1}^{2} \neq 0$, so $\delta\left(a_{1}\right) \neq 0$. We may then take $\xi=\delta\left(a_{1}\right)$. Since

$$
\xi^{2}=\delta\left(a_{1}\right) \delta\left(a_{1}\right)=\delta\left(a_{1} \Delta\left(a_{1}\right)\right)=\delta\left(a_{1}^{3}\right)=0,
$$

so $\operatorname{Res}_{G, S D} \xi=\eta$. Let $\zeta$ denote the generator of degree 4 in $H^{*}(G, \mathbb{Z})_{2}$. Since $\Delta\left(b_{3}\right)=\operatorname{Sq}^{1}\left(b_{3}\right)=0$, so $\delta\left(b_{3}\right) \in \operatorname{Ker} \pi_{*}=\operatorname{Im} 2$. Therefore $\delta\left(b_{3}\right)=2^{n-1} \zeta$. Then $H^{6}(G, \mathbb{Z})_{2}=\langle\xi \zeta, \nu\rangle$ for some $\nu$. Since $\Delta\left(e_{5}\right)=\operatorname{Sq}^{1}\left(e_{5}\right)=b_{3}^{2} \neq 0$, so $\delta\left(e_{5}\right) \neq 0$. Hence we may take $\nu=\delta\left(e_{5}\right)$. Note that

$$
\xi \nu=\delta\left(a_{1}\right) \delta\left(e_{5}\right)=\delta\left(a_{1} \Delta\left(e_{5}\right)\right)=\delta\left(a_{1} b_{3}^{2}\right)=0
$$

Let $\mu$ denote the generator of degree 9 in $H^{*}(G, \mathbb{Z})_{2}$. Since $\Delta\left(b_{3} e_{5}\right)=b_{3}\left(b_{3}^{2}\right) \neq 0$, so $\delta\left(b_{3} e_{5}\right) \neq 0$. Hence we may take $\mu=\delta\left(b_{3} e_{5}\right)$. Then

$$
\mu^{2}=\delta\left(b_{3} e_{5}\right) \delta\left(b_{3} e_{5}\right)=\delta\left(b_{3} e_{5} \Delta\left(b_{3} e_{5}\right)\right)=\delta\left(b_{3} e_{5}\left(b_{3}^{3}\right)\right)=\delta\left(b_{3}^{4} e_{5}\right)
$$

and

$$
\nu^{3}=\delta\left(e_{5}\right) \delta\left(e_{5}\right) \delta\left(e_{5}\right)=\delta\left(e_{5} b_{3}^{2}\right) \delta\left(e_{5}\right)=\delta\left(b_{3}^{4} e_{5}\right) .
$$

Therefore $\mu^{2}=\nu^{3}$. It follows that $\operatorname{Res}_{G, S D} \nu$ must $\alpha \beta$ and $\operatorname{Res}_{G, S D} \mu$ must be $\alpha \gamma$. Note that

$$
\xi \mu=\delta\left(a_{1}\right) \delta\left(b_{3} e_{5}\right)=\delta\left(a_{1}^{2} b_{3} e_{5}\right)=0
$$

By inspection, the elements $\xi, \zeta, \nu, \mu$ are sufficient to generate $H^{*}(G, \mathbb{Z})_{2}$. We thus have

Proposition 2.3. In case $2, H^{*}(G, \mathbb{Z})_{2}$ is generated by the elements

$$
\xi, \zeta, \nu, \mu
$$

where $\operatorname{deg} \xi=2, \operatorname{deg} \zeta=4, \operatorname{deg} \nu=6, \operatorname{deg} \mu=9$ subject to the relations

$$
\begin{aligned}
& 2 \xi=2^{n} \zeta=2 \nu=2 \mu=0 \\
& \xi^{2}=\xi \nu=\xi \mu=0, \quad \mu^{2}=\nu^{3} .
\end{aligned}
$$

Further, $\operatorname{Res}_{G, S D} \xi=\eta, \operatorname{Res}_{G, S D} \zeta=\alpha, \operatorname{Res}_{G, S D} \nu=\alpha \beta, \operatorname{Res}_{G, S D} \mu=\alpha \gamma$.

CASE (3). 3||$A_{G}\left(Q_{8}\right)|, 3 \nmid| A_{G}\left(C_{2} \times C_{2}\right) \mid$.

By Proposition 2.1,

$$
\begin{aligned}
P_{G}\left(H^{*}(G, \mathbb{Z})_{2} \otimes \mathbb{F}_{2}, t\right) & =\frac{1+t^{3}}{(1-t)\left(1-t^{4}\right)} \cdot \frac{t}{1+t}+\frac{1}{1+t} \\
& =\frac{1+t^{5}}{\left(1-t^{2}\right)\left(1-t^{4}\right)}
\end{aligned}
$$


We have

\begin{tabular}{|c|c|c|c|c|c|c|c|c|c|c|c|c|}
\hline$n$ & 1 & 2 & 3 & 4 & 5 & 6 & 7 & 8 & 9 & 10 & 11 & 12 \\
\hline $\operatorname{dim}_{\mathbf{F}_{2}} H^{n}(G, \mathbb{Z})_{2} \otimes \mathbb{F}_{2}$ & 0 & 1 & 0 & 2 & 1 & 2 & 1 & 3 & 2 & 3 & 2 & 4 \\
\hline
\end{tabular}

For convenience, let $a_{1}=\xi, b_{3}=\mu$ and $d_{4}=\beta$ in Proposition 1.6. Since $\Delta\left(a_{1}\right)=a_{1}^{2} \neq 0$, so $\delta\left(a_{1}\right) \neq 0$. Then $H^{2}(G, \mathbb{Z})_{2}=\langle\xi\rangle$ where $\xi=\delta\left(a_{1}\right)$. Since $\xi^{2} \neq 0$, so $H^{4}(G, \mathbb{Z})_{2}=\left\langle\xi^{2}, \zeta\right\rangle$ for some $\zeta$. Let $\nu$ denote the generator of degree 5 in $H^{*}(G, \mathbb{Z})_{2}$. Since $\Delta\left(a_{1} b_{3}\right)=a_{1}^{2} b_{3} \neq 0$, so $\delta\left(a_{1} b_{3}\right) \neq 0$. We may then take $\nu=\delta\left(a_{1} b_{3}\right)$. Clearly, $\operatorname{Res}_{G, S D} \xi=\eta+\beta, \operatorname{Res}_{G, S D} \zeta=\alpha$ and $\operatorname{Res}_{G, S D} \nu=\gamma$. Since $\gamma^{2}=\beta^{3} \alpha$, so we must have $\nu^{2}=\xi^{3} \zeta$. By inspection, the elements $\xi, \zeta$ and $\nu$ are sufficient to generate $H^{*}(G, \mathbb{Z})_{2}$. We thus have

Propos ITION 2.4. In case $3, H^{*}(G, \mathbb{Z})_{2}$ is generated by the elements

$$
\xi, \zeta, \nu
$$

where $\operatorname{deg} \xi=2, \operatorname{deg} \zeta=4, \operatorname{deg} \nu=5$ subject to the relations

$$
\begin{aligned}
& 2 \xi=2^{n} \zeta=2 \nu=0 \\
& \nu^{2}=\xi^{3} \zeta
\end{aligned}
$$

Further, $\operatorname{Res}_{G, S D} \xi=\eta+\beta, \operatorname{Res}_{G, S D} \zeta=\alpha, \operatorname{Res}_{G, S D} \nu=\gamma$.

CASE (4). $3 \nmid\left|A_{G}\left(Q_{8}\right)\right|, 3 \nmid\left|A_{G}\left(C_{2} \times C_{2}\right)\right|$.

The Poincaré series of $H^{*}(G, \mathbb{Z})_{2} \otimes \mathbb{F}_{2}$ is

$$
\begin{aligned}
P_{G}\left(H^{*}(G, \mathbb{Z})_{2} \otimes \mathbb{F}_{2}, t\right) & =\frac{1+t}{(1-t)\left(1-t^{4}\right)} \cdot \frac{t}{1+t}+\frac{1}{1+t} \\
& =\frac{1+t^{2}-t^{4}+t^{5}}{\left(1-t^{2}\right)\left(1-t^{4}\right)}
\end{aligned}
$$

We have

\begin{tabular}{|c|c|c|c|c|c|c|c|c|c|c|c|c|}
\hline$n$ & 1 & 2 & 3 & 4 & 5 & 6 & 7 & 8 & 9 & 10 & 11 & 12 \\
\hline $\operatorname{dim}_{\mathbb{F}_{2}} H^{n}(G, \mathbb{Z})_{2} \otimes \mathbb{F}_{2}$ & 0 & 2 & 0 & 2 & 1 & 3 & 1 & 3 & 2 & 4 & 2 & 4 \\
\hline
\end{tabular}

Since $\operatorname{dim}_{\mathbb{F}_{2}} H^{n}(G, \mathbb{Z})_{2} \otimes \mathbb{F}_{2}=\operatorname{dim}_{\mathbb{F}_{2}} H^{n}(S D, \mathbb{Z}) \otimes \mathbb{F}_{2}$ for $n \leqslant 5$ and $H^{*}(S D, \mathbb{Z})$ is generated by elements of degrees $\leqslant 5$, we have

PROPOSITION 2.5. In case $4, H^{*}(G, \mathbb{Z})_{2} \cong H^{*}(S D, \mathbb{Z})$. 


\section{REFERENCES}

[1] T. Asai and H. Sasaki, 'The mod 2 cohomology algebras of finite groups with dihedral Sylow 2-subgroups', Comm. Algebra 21 (1993), 2771-2790.

[2] H. Cárdenas and E. Lluis, 'On the integral cohomology of a Sylow subgroup of the symmetric group', Comm. Algebra 18 (1990), 105-134.

[3] L. Evens and S. Priddy, 'The cohomology of the semi-dihedral group', in Conference on Algebraic Topology in Honour of P. Hilton, (R. Piccinini and D. Sjerve, Editors), Contemporary Mathematics Vol. 37 (American Mathematical Society, Providence, RI, 1985), pp. 61-72.

[4] J. Martino, Stable splittings of the Sylow 2-subgroups of $S L_{3}\left(\mathbb{F}_{q}\right), q$ odd, Thesis (Northwestern University, 1988).

[5] P. Webb, 'Two classifications of simple Mackey functors with applications to group cohomology and the decomposition of classifying spaces', J. Pure Appl. Algebra 88 (1993), 265-304.

Department of Mathematics

The University of Queensland

Queensland 4072

Australia 